\title{
ATOMICAL GROTHENDIECK CATEGORIES
}

\section{NĂSTĂSESCU and B. TORRECILLAS}

\author{
Received 11 September 2002
}

\begin{abstract}
Motivated by the study of Gabriel dimension of a Grothendieck category, we introduce the concept of atomical Grothendieck category, which has only two localizing subcategories, and we give a classification of this type of Grothendieck categories.

2000 Mathematics Subject Classification: 18E15, 16 S90.
\end{abstract}

1. Introduction. Given a Grothendieck category $\mathscr{A}$, we can associate with it the lattice of all localizing categories of $\mathscr{A}$ and denote it by $\operatorname{Tors}(\mathscr{A})$. We will show (Theorem 3.3) that if $\mathscr{A}$ has Gabriel dimension, then the lattice Tors $(\mathscr{A})$ is semi-Artinian. Moreover, the Gabriel dimension of $\mathscr{A}$ is exactly the Loewy length of this lattice. Example 3.4 proves that the converse statement does not hold. (Therefore, the properties of the lattice Tors $(\mathscr{A})$ do not determine the properties of the category $\mathscr{A}$.) These facts suggest introducing the concept of atomical Grothendieck category. Precisely, $\mathscr{A}$ will be called atomical if the lattice Tors $(\mathscr{A})$ has only two elements, that is, $\mathscr{A}$ has only two localizing subcategories, namely, $\{0\}$ and $\mathscr{A}$. The classification of atomical Grothendieck categories is given in Section 4.

2. Preliminaries. Throughout this paper, $\mathscr{A}$ will denote a Grothendieck category, that is, an abelian category with a generator, such that colimits exist and direct limits are exact.

It is well known that in a Grothendieck category each object $X$ has an injective hull, denoted in the sequel by $E(X)$.

If $\mathscr{A}$ is a category, then by a subcategory $\mathscr{B}$ of $\mathscr{A}$ we will always mean a full subcategory of $\mathscr{A}$.

A subcategory $\mathscr{C}$ of $\mathscr{A}$ is called closed (or hereditary pretorsion class) if it is closed with respect to kernels, cokernels, and direct sums.

By $\sigma[X]$ we denote the full subcategory of $\mathscr{A}$ whose objects are subobjects of $X$-generated objects. These objects are said to be subgenerated by $X$, and $X$ is a subgenerator of $\sigma[X]$. This is the smallest closed full subcategory of $\mathscr{A}$ containing $X$.

By definition, the objects of $\sigma[X]$ form a closed subcategory in $\mathscr{A}$. On the other hand, every closed subcategory $\mathscr{T}$ in $\mathscr{A}$ is of the form $\sigma[X]$ for some object $X$; for example, for $X$ equals the direct sum of all (nonisomorphic) cyclic objects in $\mathcal{T}$. 
Following Goldman [2], a functor $\tau: \mathscr{A} \rightarrow \mathscr{A}$ is called a kernel functor if

(1) it is a subfunctor of the identity functor, that is, $\tau(M) \subseteq M$ and $f: M \rightarrow$ $M^{\prime}$ implies $f(\tau(M)) \subseteq \tau\left(M^{\prime}\right)$;

(2) $N \subseteq M$ implies $\tau(N)=N \cap \tau(M)$.

The trivial kernel functors 0 and $\infty$ are defined by setting $0(X)=0$, and $\infty(X)=$ $X$, for every object $X \in \mathscr{A}$.

For any kernel functor $\tau, X$ is called a $\tau$-torsion module if $\tau(X)=X$, and a $\tau$-torsion-free module if $\tau(X)=0$. The collection of $\mathscr{T}_{\tau}$ of all the $\tau$-torsion module is a closed subcategory of $\mathscr{A}$. Conversely, for any closed subcategory $\mathscr{C}$, there exists a unique kernel functor $\tau$ such that $\mathscr{C}=\mathcal{T}_{\tau}$.

LEMMA 2.1. Let $G$ be a generator of $A$ and $\mathscr{C}$ a closed subcategory. Then

$$
\mathscr{C}=\sigma[\oplus\{G / X \mid G / X \in \mathscr{b}\}] .
$$

COROLLARY 2.2. The closed subcategories (and hence, the kernel functors) form a set.

Proposition 2.3. The set of all closed subcategories of $A$ is a complete lattice. For a family $\left\{X_{\lambda}\right\}_{\Lambda}$ of objects of $\mathscr{A}$,

$$
\begin{aligned}
& \bigvee_{\Lambda} \sigma\left[X_{\lambda}\right]=\sigma\left[\oplus_{\Lambda} X_{\lambda}\right], \\
& \bigwedge_{\Lambda} \sigma\left[X_{\lambda}\right]=\bigcap_{\Lambda} \sigma\left[X_{\lambda}\right] .
\end{aligned}
$$

REMARK 2.4. (1) (cf. [4]). For a coalgebra $C$, the lattice of all closed subcategories of the category of comodules over $C$ is anti-isomorphic to the lattice of subcoalgebras of $C$.

(2) The Serre subcategories of $\mathscr{A}$ (i.e., the subcategories $\mathscr{Y}$ of $\mathscr{A}$ satisfying that for any exact sequence from $\mathscr{A}$,

$$
0 \longrightarrow X^{\prime \prime} \longrightarrow X \rightarrow X^{\prime} \longrightarrow 0
$$

where $X$ is in $\mathscr{Y}$ if and only if $X^{\prime}$ and $X^{\prime \prime}$ are in $\mathscr{Y}$ ) do not form a set. For example, we consider the Grothendieck category $\mathscr{V}$ of vector spaces over a division ring. For any cardinal $\alpha$, the subcategory of all vector spaces of dimension less than or equal to $\alpha$ is a Serre subcategory. Thus, the Serre subcategories of $\mathscr{V}$ are not a set.

We now recall the notion of semi-Artinian lattice. Let $L$ be an upper continuous and modular lattice. An atom of $L$ is a nonzero element $a \in L$ such that whenever $b \in L$ and $b<a$, then $b=0$, that is, the interval $[0, a]$ has exactly two elements, 0 and $a$. If $a, b \in L$ and $x<y$, then the interval $[x, y]$ is simple if $y$ is an atom in the sublattice $[x, y]$ of $L$. The lattice is called semiatomic if 1 is a joint of atoms, and $L$ is called semi-Artinian if for every $x \in L, x \neq 1$, the sublattice $[x, 1]$ of $L$ contains an atom. 
The (ascending) Loewy series of $L$,

$$
s_{0}(L)<s_{1}(L)<\cdots<s_{\lambda(L)}(L),
$$

is defined recursively as follows: $s_{0}(L)=0, s_{1}(L)$ is the socle $\operatorname{Soc}(L)$ of $L$ (i.e., the join of all atoms of $L$ ), and if the elements $s_{\beta}(L)$ of $L$ have been defined for all ordinals $\beta<\alpha$, then $s_{\alpha}(L)=\bigvee_{\beta<\alpha} s_{\beta}(L)$ if $\alpha$ is a limit ordinal and $s_{\alpha}=$ $\operatorname{Soc}\left(\left[s_{\gamma}(L), 1\right]\right)$ if $\alpha=\gamma+1$.

The Loewy length $\lambda(L)$ of $L$ is the least ordinal such that $s_{\lambda}(L)=s_{\lambda+1}(L)$.

3. Gabriel dimension and localizing subcategories. A subcategory $\mathscr{T} \subseteq \mathscr{A}$ is a localizing subcategory if it is closed under subobjects, quotient objects, extensions, and coproducts. If $\mathscr{B} \subseteq \mathscr{A}$ is an arbitrary subcategory, we denote by $\mathscr{T}(\mathscr{B})$ the smallest localizing subcategory containing $\mathscr{B}$.

EXAMPLES 3.1. (i) An object $A \in A$ is singular if there exists a short exact sequence

$$
0 \longrightarrow A^{\prime \prime} \longrightarrow A^{\prime} \longrightarrow A \longrightarrow 0
$$

where the monomorphism is essential.

In any Grothendieck category, we can always consider the Goldie localizing subcategory, denoted by $\mathscr{G}$, as the smallest localizing subcategory containing the singular objects.

(ii) We can associate to any injective object $E \in \mathscr{A}$ a localizing subcategory

$$
\mathscr{T}_{E}=\left\{A \in \mathscr{A} \mid \operatorname{Hom}_{\mathscr{A}}(A, E)=0\right\} .
$$

This localizing subcategory is said to be cogenerated by $E$.

(iii) For a projective object $P \in \mathscr{A}$, we can define

$$
\mathscr{T}_{P}=\left\{A \in \mathscr{A} \mid \operatorname{Hom}_{\mathscr{A}}(P, A)=0\right\} .
$$

It is clear that $\mathscr{T}_{P}$ is a localizing subcategory closed under direct product.

(iv) If $S$ is a simple object in $\mathscr{A}$, we denote by $\mathscr{A}_{S}$ the smallest localizing subcategory containing $S$. In fact,

$$
\mathscr{A}_{S}=\{M \in \mathscr{A} \mid N \subset M, M / N \text { contains a simple object isomorphic to } S\} \text {. }
$$

The objects in this localizing subcategory are called $S$-primary.

Let $\mathscr{T}$ be a localizing subcategory. The corresponding torsion functor or idempotent kernel functor is denoted by

$$
t_{\mathscr{T}}: \mathscr{A} \longrightarrow \mathscr{T}
$$

This functor assigns to an object $A \in \mathscr{A}$ the maximal subobject $t_{\mathscr{T}}(A) \subseteq A$ in $\mathscr{T}$. An object $X \in \mathscr{A}$ is $\mathscr{T}$-torsion-free (resp., $\mathscr{T}$-torsion) if $t_{\mathscr{T}}(X)=0$ (resp., 
$\left.t_{\mathscr{T}}(X)=X\right)$. Let $H^{1} t_{\mathscr{T}}$ denote the first higher derived functor of the left exact functor $t_{\mathscr{T}}$. A $\mathscr{T}$-torsion-free object $E \in \mathscr{A}$ is $\mathscr{T}$-closed if $H^{1} t_{\mathscr{T}}=0$.

If $\mathcal{T}$ is a localizing subcategory of $\mathscr{A}$, we can consider the quotient category $\mathscr{A} / \mathcal{T}$. We denote by $T_{\mathscr{T}}: \mathscr{A} \rightarrow \mathscr{A} / \mathcal{T}$ the canonical functor and by $S_{\mathscr{T}}: \mathscr{A} / \mathscr{T} \rightarrow \mathscr{A}$ the right adjoint functor of $T_{\mathscr{T}}$.

It is well known that the category $\mathscr{A} / \mathcal{T}$ is equivalent to the full subcategory of $\mathscr{A}$ of $\mathscr{T}$-closed objects.

It is well known that $\mathscr{A}$ has a set of localizing subcategories Tors $(\mathscr{A})$. Given a family of localizing subcategories $\left(\mathscr{C}_{i}\right)_{i \in I}$, we define the meet by $\bigwedge_{i \in I} \mathscr{b}_{i}=$ $\bigcap_{i \in I} \mathscr{C}_{i}$, and the join by $\bigvee_{i \in I} \mathscr{C}_{i}$, as the smallest localizing subcategory containing the union of the $\mathscr{C}_{i}$. Notice that $\operatorname{Tors}(\mathscr{A})$ is not a sublattice of the lattice of all closed subcategories of $\mathscr{A}$. It is also known that this set is a frame (i.e., it is a complete lattice $L$ such that $a \wedge(\bigvee X)=\bigvee\{a \wedge x \mid x \in X\}$ for each element $a$ and subset $X$ of $L$ ). Frames are also known as local lattices, complete Heyting algebras, or complete Brouwerian lattices. The lattice of closed subcategories is not a frame in general.

We need the following preliminary result.

Proposition 3.2. Let $\mathscr{A}$ be a Grothendieck category and let $\mathscr{b} \subseteq \mathscr{A}$ be a localizing subcategory. There exists a bijective correspondence between the localizing subcategories of $\mathscr{A} / \mathscr{C}$ and the localizing subcategories $\mathscr{B}$ of $\mathscr{A}$ containing C. Moreover, Tors $(\mathscr{A} / \mathscr{C})$ is a subframe of $\operatorname{Tors}(\mathscr{A})$

Proof. Let $T: A \rightarrow A / \mathscr{C}$ be the canonical functor. Consider $\mathscr{B}$, a localizing subcategory of $\mathscr{A}$ containing $\mathscr{C}$, then $T(\mathscr{B})=\{Z \in \mathscr{A} / \mathscr{C} \mid Z \cong T(X), X \in \mathscr{B}\}$ is a localizing subcategory of $\mathscr{A} / \mathscr{C}$. In fact, it is clear that $T(\mathscr{B})$ is closed under subobjects, quotients, and direct sums. It remains to show that $T(\mathscr{B})$ is closed under extensions. First, we observe that $T(\mathscr{B})=\{Z \in \mathscr{A} / \mathscr{C} \mid S(Z) \in \mathscr{B}\}$. To see this, consider the exact sequence

$$
0 \longrightarrow \operatorname{Ker} f \longrightarrow X \longrightarrow S T(X) \cong S(Z) \longrightarrow \operatorname{Coker} f \longrightarrow 0
$$

where $\operatorname{Ker} f$, Coker $f \in \mathscr{C}$. Therefore, $\operatorname{Ker} f$, Coker $f \in \mathscr{B}$, and $X \in \mathscr{B}$ if and only if $S(Z) \in \mathscr{B}$. Now if

$$
0 \longrightarrow Z^{\prime} \longrightarrow Z \longrightarrow Z^{\prime \prime} \longrightarrow 0
$$

is an exact sequence in $\mathscr{A} / \mathscr{C}$, with $Z^{\prime}, Z^{\prime \prime} \in T(\mathscr{B})$, we apply the functor $S$ to obtain

$$
0 \longrightarrow S\left(Z^{\prime}\right) \longrightarrow S(Z) \longrightarrow S\left(Z^{\prime \prime}\right)
$$

Thus, $S(Z) \in \mathscr{B}$ and $Z \in T(\mathscr{B})$.

Let $\mathscr{D}$ be a localizing subcategory in $\mathscr{A} / \mathscr{C}$. we define $T^{-1} \mathscr{D}=\{X \in \mathscr{A} \mid T(X) \in$ $\mathscr{D}\}$. Since $T$ is an exact functor which commutes with direct sums, then $T^{-1}(\mathscr{D})$ is a localizing subcategory which contains $\mathscr{b}$. It is not difficult to see that these 
two operations establish a bijection between the localizing subcategories of $\mathscr{A} / \mathscr{C}$ and the localizing subcategories $\mathscr{B}$ of $\mathscr{A}$ containing $\mathscr{C}$.

We now recall the notion of Gabriel dimension of a Grothendieck category $\mathcal{A}$. For any ordinal $\alpha$, we will denote by $\mathscr{C}_{\alpha}$ the localizing subcategory defined in the following way: $\mathscr{C}_{0}$ is the zero subcategory; $\mathscr{C}_{1}$ is the smallest localizing subcategory containing all simple objects; if $\alpha=\beta+1$, an object $X$ of $\mathscr{A}$ will be contained in $\mathscr{C}_{\alpha}$ if and only if $T_{\beta}(X) \in O b\left(\mathscr{A} / \mathscr{C}_{\beta}\right)_{1}$, where $T_{\beta}: \mathscr{A} \rightarrow \mathscr{A} / \mathscr{C}_{\beta}$ is the canonical functor; and if $\alpha$ is a limit ordinal, then $\mathscr{C}_{\alpha}$ is the localizing subcategory generated by all localizing subcategories $\mathscr{C}_{\beta}$, with $\beta \leq \alpha$.

It is clear that if $\alpha \leq \alpha^{\prime}$, then $\mathscr{C}_{\alpha} \subseteq \mathscr{C}_{\alpha^{\prime}}$. Hence, there exists an ordinal $\tau$ such that $\mathscr{C}_{\tau}=\mathscr{C}_{\alpha}$ for any ordinal $\alpha \geq \tau$. We define $\mathscr{C}_{\tau}=\cup_{\alpha} C_{\alpha}$.

The set of localizing subcategories $\mathscr{C}_{\alpha}$ is called the Gabriel filtration of $\mathscr{A}$. We say that an object $X$ of $\mathscr{A}$ has Gabriel dimension if $X$ is in $\mathscr{C}_{T}$. Then the smallest ordinal $\alpha$ verifying $X$ in $\mathscr{C}_{\alpha}$ is called the Gabriel dimension of $X$.

We say that $\mathscr{A}$ has Gabriel dimension if $\mathscr{A}=\mathscr{C}_{\tau}$ or, equivalently, any object of $\mathscr{A}$ has Gabriel dimension. We are now ready for the main result of this section.

THEOREM 3.3. Let $A$ be Grothendieck category. If $A$ has Gabriel dimension $\alpha$, then $\operatorname{Tors}(\mathscr{A})$ is a semi-Artinian lattice with Loewy length $\alpha$.

Proof. We will show this result by transfinite induction. If $G$ - $\operatorname{dim} \mathscr{A}=1$, then $\mathscr{A}=\mathscr{C}_{1}$, the localizing subcategory generated by the simple objects of $\mathscr{A}$. Hence, $\mathscr{A}=\bigvee \mathscr{C}_{S}$.

Now, we assume that the result is true for any Grothendieck category of Gabriel dimension $\beta<\alpha$. If $\alpha=\gamma+1$ is not a limit ordinal, then any object $X \in$ $\mathscr{A}$ belongs to $\mathscr{C}_{\alpha}$ or, equivalently, $T_{\gamma}(X) \in\left(\mathscr{A} / \mathscr{C}_{\gamma}\right)_{1}$. Now, $\mathscr{C}_{\gamma}=s_{\gamma}\left(\operatorname{Tors}\left(\mathscr{C}_{\gamma}\right)\right)$. If $X \in \mathscr{A}$ satisfies that $T_{\gamma}(X)$ is a simple object in $\mathscr{A} / \mathscr{C}_{\gamma}$, then $\left(\mathscr{A} / \mathscr{C}_{\gamma}\right)_{T_{\gamma}(X)}$ is an atom in Tors $\left(\mathscr{A} / \mathscr{C}_{\gamma}\right)$. By Proposition 3.2, $T_{\gamma}^{-1}\left(\left(\mathscr{A} / \mathscr{C}_{\gamma}\right)_{T_{\gamma}(X)}\right)$ is an atom in $\left[\mathscr{C}_{\gamma}, \mathscr{A}\right]$. We will see that $\mathscr{A}=\bigvee T_{\gamma}^{-1}\left(\mathscr{A} / \mathscr{C}_{\gamma}\right)_{T_{\gamma}(X)}$. Let $A \in \mathscr{A}$ and consider $A \rightarrow$ $A^{\prime} \rightarrow 0$, with $A^{\prime} \neq 0$. Applying the functor $T_{\gamma}$, we obtain $T_{\gamma}(A) \rightarrow T_{\gamma}\left(A^{\prime}\right) \rightarrow 0$. If $T\left(A^{\prime}\right)=0$, the proof is finished; otherwise $T_{\gamma}\left(A^{\prime}\right)$ contains a simple object $T_{\gamma}(X)$. Therefore, we have $K \rightarrow X \rightarrow A^{\prime}$ and $A^{\prime}$ contains $X / K$ which is in $T_{\gamma}^{-1}\left(\mathscr{A} / \mathscr{C}_{\gamma}\right)_{T_{\gamma}(X)}$. If $\alpha$ is a limit ordinal $\mathscr{A}=\bigcup_{\beta<\alpha} \mathscr{C}_{\beta}$, then $\mathscr{A}=\bigvee_{\beta<\alpha} \mathscr{C}_{\beta}$.

The next example shows that the converse of Theorem 3.3 is not true.

EXAMPLE 3.4. Let $R$ be a commutative nondiscrete valuation domain of Krull dimension 1, with maximal ideal $M$. Then

(i) $M^{2}=M$,

(ii) if $x \in M$, then $\bigcap_{n \geq 0} R x^{n}=0$,

(iii) $\operatorname{Tors}(R$-Mod) has four elements:

$$
\{0\} \subseteq(R-\operatorname{Mod})_{R / M} \subseteq \mathcal{T} \subseteq R-\operatorname{Mod},
$$

where $\mathscr{T}$ is the usual torsion theory in a domain and $(R \text {-Mod })_{R / M}$ is a semisimple category, 
(iv) the quotient category $R$-Mod $/(R \text {-Mod })_{R / M}$ has no simple objects,

(v) $R$-Mod has no Gabriel dimension.

Proof. (i) Take $x \in M$. Since the valuation is not discrete, we can find an element $y \in M$ such that $v\left(y^{2}\right)=2 v(y)<v(x)$. Hence, $x \in\left(y^{2}\right) \subseteq M^{2}$ and $M=M^{2}$.

(ii) Let $2=\bigcap_{n \geq 0} R x^{n}$. We claim that 2 is a prime ideal. Let $a, b \in R$ with $a \notin 2$ and $b \notin 2$. Hence, there exist $n$ and $m$ such that $a \notin R x^{n}$ and $b \notin R x^{m}$. Thus, $R x^{n} \subset R a$ and $R x^{m} \subset R b$. Then $R x^{n+m} \subset R x^{n} b \subset R a b$ and $a b \notin 2$. Therefore, $2=0$.

(iii) Let $\mathscr{C}$ be a localizing subcategory properly containing $(R \text {-Mod })_{R / M}$ and let $I$ be a nonzero ideal. We take $J \varsubsetneqq M$ with $R / J \in \mathscr{C}$. Thus, there exist $x \in M \backslash J$. By (ii), $\bigcap_{n \geq 0} R x^{n}=0$, and it follows that $R x^{n} \subseteq I$ for some $n$ and $R / I \in \mathscr{C}$.

(iv) Any simple object of $R$-Mod $/(R-\operatorname{Mod})_{R / M}$ is given by an $(R-\operatorname{Mod})_{R / M^{-}}$ critical ideal, but this kind of ideals is prime. This prime is 0 . So the cocritical module is isomorphic to $R$. Therefore, $R / I$ is semisimple for every nonzero ideal $I$ of $R-$ a contradiction.

(v) The proof follows from (iv).

4. Atomical Grothendieck categories. We have proved in Theorem 3.3 that if a Grothendieck category has Gabriel dimension, then the lattice of localizing subcategories is semi-Artinian. Example 3.4 shows that the converse is not true. This fact suggests the study of Grothendieck categories $\mathscr{A}$ with the property that the category $\mathscr{A}$ is an atom in the lattice Tors $(\mathscr{A})$, that is, $\mathscr{A}$ has only two localizing subcategories $\{0\}$ and $\mathscr{A}$.

DEFINITION 4.1. A Grothendieck category $\mathscr{A}$ is called atomical if it has only two localizing subcategories, namely, $\{0\}$ and $\mathscr{A}$.

A maximal localizing category $\mathscr{T}$ is a maximal element of $\operatorname{Tors}(\mathscr{A})-\mathscr{A}$. By Proposition $3.2, \mathscr{A}$ is a maximal localizing category of $\mathscr{A}$ if and only if $\mathscr{A} / \mathscr{T}$ is an atomical Grothendieck category.

Recall that an object $C$ in $\mathscr{A}$ is called a cogenerator if for each nonzero morphism $f: X \rightarrow Y$ in $\mathscr{A}$, there exists a morphism $g: Y \rightarrow C$ such that $g f \neq 0$. This is equivalent to the existence of a monomorphism $A \rightarrow C^{I}$ for some index set $I$, for every object $A \in \mathscr{A}$. It is clear that an injective object $E$ of $\mathscr{A}$ is a cogenerator if and only if for each nonzero object $A \in \mathscr{A}$, there exists a nonzero morphism $f: A \rightarrow E$.

Proposition 4.2. If $\mathscr{A}$ is a Grothendieck category, then $\mathscr{A}$ is an atomical category if and only if every nonzero injective object of $A$ is a cogenerator.

Moreover, if the category has enough projectives, then $A$ is an atomical category if and only if every nonzero projective object of $\mathscr{A}$ is a generator.

Proof. Assume that $\mathscr{A}$ is atomical, then any nonzero injective object cogenerates a nonzero torsion-free class. Hence, this torsion-free class must be 
the whole category and this injective is a cogenerator. Since any localizing subcategory of $\mathscr{A}$ is cogenerated by an injective object, the converse is clear.

It is clear that for an atomical Grothendieck category $\mathscr{A}$, we have that the Goldie torsion theory has to be either $\{0\}$ or $\mathscr{A}$. In the first case, we say that $\mathscr{A}$ is a nonsingular Grothendieck category and we characterize this type of simple Grothendieck categories. Recall that a Grothendieck category $\mathscr{A}$ is called spectral if any short exact sequence splits and a spectral Grothendieck category is called discrete if every object is semisimple.

Proposition 4.3. Let $A$ be a Grothendieck category. The category $A$ is nonsingular atomical if and only if $\mathscr{A}$ is a spectral category which is equivalent to $R$-Mod $/ G$, where $R$ is a regular prime self-injective ring and $\varphi$ is the Goldie localizing subcategory. Moreover, A contains a simple object if and only if $R$ is isomorphic to the ring of all linear transformations of a left vector space over a division ring.

Proof. Suppose $\mathscr{A}$ is nonsingular and atomical. Since $\mathscr{A}$ is nonsingular, then $\varphi=0$. Hence, $X \subseteq^{\prime} E(X)$ with $E(X) / X$ singular, a matter which implies that $X=E(X)$ and any object is injective. Thus, $\mathscr{A}$ is a spectral Grothendieck category.

Let $U$ be a generator of $\mathscr{A}$ and $R=\operatorname{Hom}_{\mathscr{A}}(U, U)$, by the Gabriel-Oberst theorem [5, Chapter XII, Theorem 1.3] $\mathscr{A}$ is equivalent to $R$-Mod/G, where $R$ is a regular self-injective ring and $\mathscr{G}$ is the Goldie's localizing subcategory. Since $R$-Mod $/ \mathscr{G}$ is atomical, then $\mathscr{G}$ is maximal. Hence, by [1, Theorem 2.2], $0=t_{\mathscr{G}}(R)$ is a prime ideal.

Conversely, assume that $R$ is a prime self-injective regular ring. Since $R$ is prime, then it is nonsingular. Thus, $t_{\mathscr{G}}(R)=0$ is a prime ideal, where $\mathscr{G}$ is a maximal localizing subcategory by [1, Theorem 2.2]. Therefore, $R$-Mod $/ \mathscr{G}$ is an atomical Grothendieck category.

Assume that $\mathscr{A}$ contains a simple object, then $\mathscr{A}$ coincides with the localizing subcategory generated by this simple object. Hence, as an object in $R$-Mod $/ \mathscr{G}$, $R$ contains a simple object. Therefore, there exists a $\mathscr{G}$-cocritical left ideal $C$ of $R$. If $C$ is not simple as a left $R$-module, then we can find a finitely generated left ideal $I \neq 0$ contained in $C$. Since $R$ is regular, there exists a left ideal $J$ such that $I \oplus J=R$. Thus, $C=I \oplus(J \cap C)$, which is a contradiction since $I$ is essential in $C$. Therefore, $C$ is a simple left ideal and $\operatorname{Soc}(R) \neq 0$. By [3, Theorem 9.12], $R$ is the ring of all linear transformations of any left vector space over a division ring.

Conversely, if $R$ is the ring of all linear transformations of any left vector space over a division ring, then $\operatorname{Soc}(R)$ is not zero. Any simple left ideal will produce a simple object in the quotient category.

We will now consider the case where the Goldie torsion theory coincides with the whole category. When the Grothendieck category contains simple objects, 
we have the following characterization. Recall that a Grothendieck category $\mathscr{A}$ is called semi-Artinian if every nonzero object of $\mathscr{A}$ contains a simple object.

Proposition 4.4. Let $\mathscr{A}$ be a singular Grothendieck category. If $\mathscr{A}$ is atomical, and it has simple objects, then $\mathscr{A}$ is a semi-Artinian Grothendieck category with a unique isomorphic class of simple objects.

Proof. Since $\mathscr{A}$ is atomical, the localizing subcategory generated by a simple object coincides with category $\mathscr{A}$. Hence, the result follows.

Proposition 4.5. Let $\mathscr{A}$ be a locally finitely generated Grothendieck category. Then $\mathscr{A}$ is atomical if and only if any object of $\mathscr{A}$ is $S$-primary, and $\mathscr{A}$ is semisimple or singular.

We now specialize our discussion to the module category $R$-Mod. In this case, we have the following result.

Proposition 4.6. $R$-Mod is an atomical category if and only if the ring $R$ is local right perfect.

Proof. If $R$ is local right perfect, then $R$-Mod is clearly atomical. Conversely, if $R$-Mod is atomical and $R$ is nonsingular, then the Goldie torsion theory is trivial. Hence, any module is injective and $R$ is semisimple. Since there is only an isomorphic class of simple modules, $R$ is simple Artinian. We only need to consider the case when $R$ is singular. But then $R$-Mod $=(R \text {-Mod })_{S}$ for some simple left $R$-module $S$ and there is only an isomorphic class of left simple $R$-modules. Thus, $R$ is semi-Artinian and $J=\operatorname{ann}(S)$. We will see that $R / J$ is a simple Artinian ring. In fact, consider $\operatorname{Soc}(R / J)=A / J \neq 0$. If $A \neq R$, then $A \subseteq M$ for some maximal left ideal $M$. Therefore, $A(R / M)=0$ and $A \subseteq \operatorname{ann}(S)=J$, a contradiction. Hence, $A=R$ and $R / J$ is simple Artinian. Since $R$ is semi-Artinian, $J$ is $T$-nilpotent. Since $R / J$ is simple Artinian and $J$ is $T$-nilpotent, then $R$ is a local right perfect ring.

Now, we consider the case of closed subcategories of $R$-Mod.

COROLLARY 4.7. Let $M$ be a left R-module. Then $\sigma[M]$ is an atomical category if and only if either $M$ is semisimple or $M$ is $S$-primary with $S$ a simple singular left $R$-module.

Finally, we present an example of a singular atomical Grothendieck category without simple objects.

EXAMPLE 4.8. We consider the same ring as in Example 3.4. Then the quotient category $\mathscr{T} /(R \text {-Mod })_{(R / M)}$ is an atomical singular Grothendieck category without simple objects.

Proof. We have proved that $R$-Mod $/(R \text {-Mod })_{(R / M)}$ has no simple objects, then $\mathscr{T} /(R \text {-Mod })_{(R / M)}$ has no simple objects. We also know from Example 3.4 that this category is atomical. We will denote by $T: \mathscr{T} \rightarrow \mathscr{T} /(R-\operatorname{Mod})_{(R / M)}$ the 
canonical functor. Let $0 \neq I \subset M$ be an ideal of $R$ such that $I \neq M$. It is clear that $R / I \in \mathcal{T}$, and $R / I \notin(R \text {-Mod })_{(R / M)}$. Let $J / I$ be the torsion part of $R / I \in$ $(R \text {-Mod })_{(R / M)}$. Since $M^{2}=M$, then $J \subset M$ and $J \neq M$. By the exact sequence

$$
0 \longrightarrow J / I \longrightarrow R / I \longrightarrow R / J \longrightarrow 0
$$

it follows that $T(R / I) \simeq T(R / J)$. Since $R$ is a valuation ring, we have that $R / J$ is a uniform (coirreducible) $R$-module, so $T(R / J)$ is still uniform in the quotient category. Denote $X=T(R / J) \simeq T(R / I)$. Then $X$ is uniform and contains no simple objects (because the category does not have nonzero simple objects). Then we can consider $Y$ as a nonzero subobject of $X$ such that $Y \neq X$. It is clear that $X / Y$ belongs to the Goldie torsion theory (of the quotient category) and $X / Y \neq 0$. As the quotient category is an atomical category, it must be the same as the Goldie torsion theory.

ACKNOWLEDGMENT. The research of the second author was partially supported by Grant BFM2002-02717 from MCT.

\section{REFERENCES}

[1] J. A. Beachy, On maximal torsion radicals, Canad. J. Math. 25 (1973), 712-726.

[2] O. Goldman, Rings and modules of quotients, J. Algebra 13 (1969), 10-47.

[3] K. R. Goodearl, Von Neumann Regular Rings, Monographs and Studies in Mathematics, vol. 4, Pitman, Massachusetts, 1979.

[4] C. Năstăsescu and B. Torrecillas, Torsion theories for coalgebras, J. Pure Appl. Algebra 97 (1994), no. 2, 203-220.

[5] B. Stenström, Rings of Quotients, An Introduction to Methods of Ring Theory, vol. 217, Springer-Verlag, New York, 1975.

C. Năstăsescu: Faculty of Mathematics, University of Bucharest, RO 70109 Bucharest 1, Romania

E-mail address: cnastase@a1 . math . unibuc. ro

B. Torrecillas: Departmento de Álgebra y Análisis Matemático, Universidad de Almería, 04071 Almería, Spain

E-mail address: btorreci@ual.es 


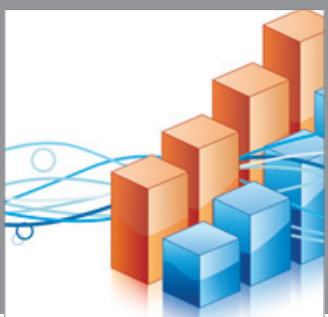

Advances in

Operations Research

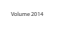

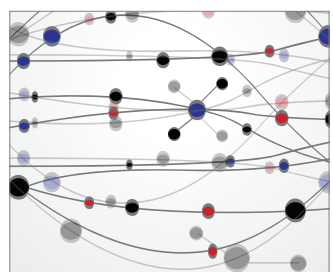

\section{The Scientific} World Journal
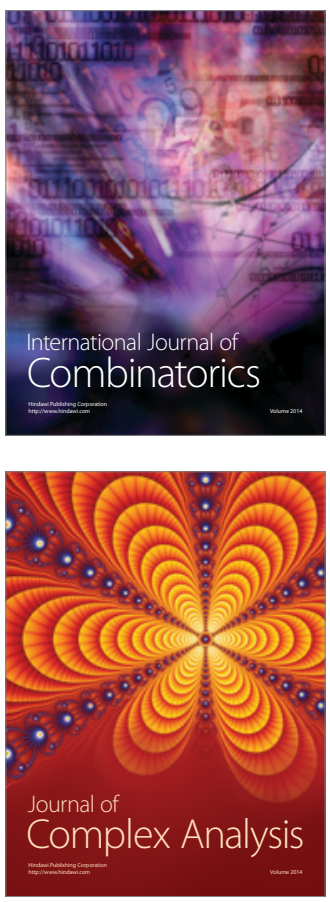

International Journal of

Mathematics and

Mathematical

Sciences
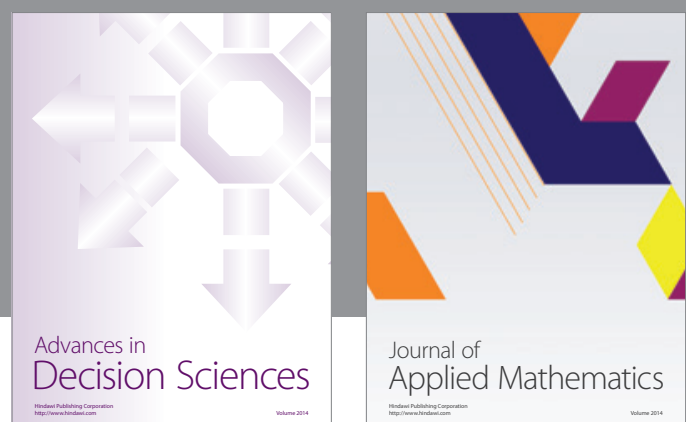

Journal of

Applied Mathematics
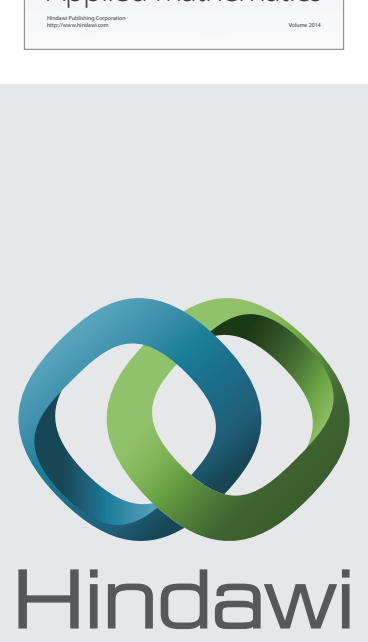

Submit your manuscripts at http://www.hindawi.com
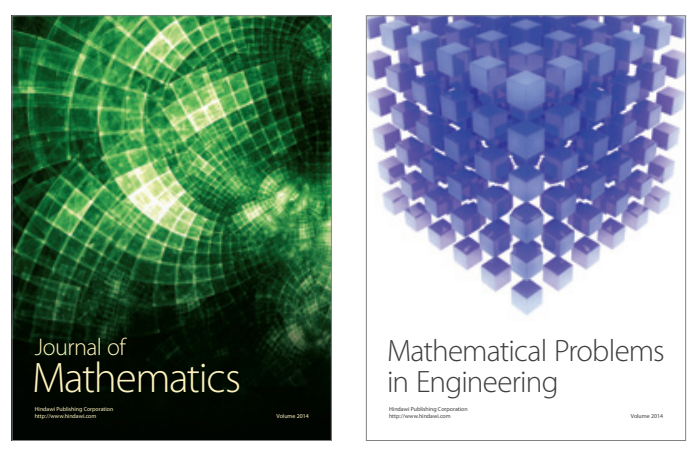

Mathematical Problems in Engineering
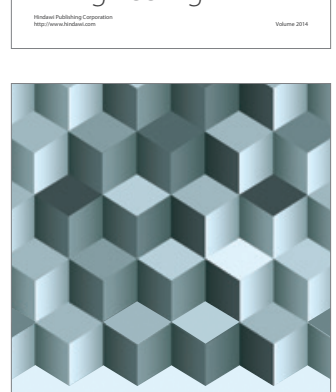

Journal of

Function Spaces
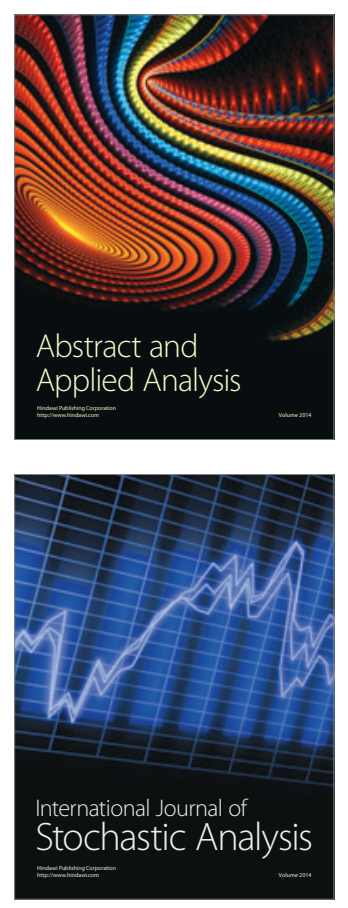

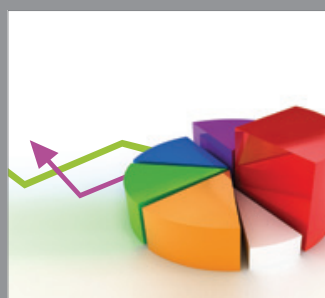

ournal of

Probability and Statistics

Promensencen
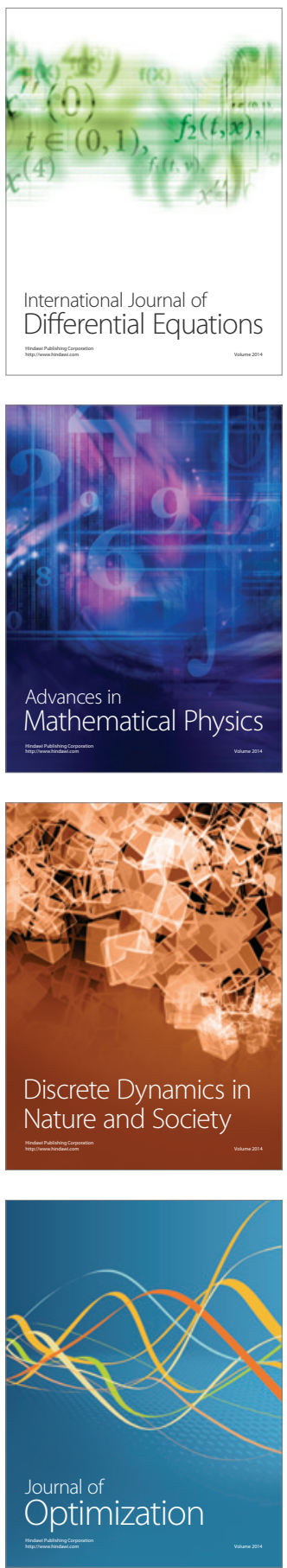